\title{
Approche analogique et réalités des phytohormones : des retards et des erreurs stratégiques?
}

Manuscrit reçu le 26 août 2016 et accepté le 3 novembre 2016

\author{
Thomas GASPAR ${ }^{1}$, Claude PENEL ${ }^{2}$ \& Hubert GREPPIN ${ }^{3}$
}

${ }^{1}$ Hormonologie Fondamentale et Appliquée et Biotechnologie Végétale, Université de Liège, Institut de Botanique B22, Sart Tilman, BE-4000 Liège, Belgique, Th.Gaspar@skynet.be

${ }^{2}$ Département de Biologie Végétale, Université de Genève, chemin Henri-Schmitt 4, CH-1218

Le Grand Saconnex, Suisse, cpenel@bluewin.ch

${ }^{3}$ Département de Biologie Végétale, Université de Genève, Quai Ernest-Ansermet 30, CH-

1211 Genève 4, Suisse, hubert.greppin@unige.ch

\section{Résumé}

Les premiers botanistes anatomistes n'avaient pu décrire de glandes endocrines à l'image de celles observées et fonctionnelles dans des organismes animaux, disposant d'un système sanguin irriguant tous leurs organes. Le concept d'hormone végétale n'émergea qu'avec les recherches sur les tropismes et la perceptibilité particulière des apex (aussi bien caulinaires que racinaires), aussi avec la découverte de la distribution polarisée, et des croissances sous-jacentes engendrées, d'où le terme premier d'hormone (sinon de substance) de croissance. L'agriculture se mit à rechercher des composés synthétiques en quête d'activités contrôlées... et plus efficaces, et en trouva.

L'application externe des unes et des autres sur différents organes sous diverses conditions expérimentales aboutit progressivement à plusieurs découvertes et conclusions importantes pour la suite des recherches.

Une même substance peut avoir des effets positifs et négatifs sur la croissance différant en importance selon la supposée "sensibilité" des organes traités, mais surtout selon les concentrations utilisées. Dans la partie "inhibition" des courbes dose-réponse caractéristiques, on parla de concentrations "sus-optimales" atteintes de l'hormone en question, mais rares furent ceux qui vérifièrent que la dite hormone appliquée pénétrait bien comme telle sans être modifiée, pour venir grossir le pool endogène; on se rendit notamment compte plus tard que la plante réagit à des applications qualifiées de sus-optimales d'agents extérieurs par une adaptation de ses systèmes cataboliques (les auxine-oxydases sont les premières en cause), sans se poser la question du devenir et des effets des produits générés; d'où l'ingénierie chimique synthétisant des homologues moins "attaquables" par les systèmes naturels de régulation (nos dérivés séléniés entre autres vraisemblablement). Les effets "rhizogènes" d'une auxine naturelle ou synthétique furent rapidement mis en évidence. Mais une hormone de croissance pouvait-elle seule provoquer un type de développement spécifique? D'où le concept de l'hormone de développement (en l'occurrence la rhizocaline) sous forme d'un complexe auxine + un sucre, des phénols, une oxydase, ...). Il en fut de même plus tard avec le "florigène" à plusieurs composants dont la gibbérelline, une autre hormone de croissance découverte entretemps. L'évidence des cytokinines (importantes celles-là pour la division cellulaire, plutôt que pour l'élongation favorisée par les auxines) mais surtout le rôle joué par la "balance" entre ces deux dernières conduisit à des avancées nouvelles et modifia aussi le concept d'hormone végétale de croissance: chaque type d'hormone pouvait être synthétisé dans tout type de cellule vivante; des gradients des différentes hormones, 
instaurés sous l'effet des conditions environnementales tout le long de la plante, sont vraisemblablement plus déterminants dans le comportement de la plante qu'une seule hormone dans l'orientation de leur croissance et de leur développement. C'est que, même les botanistes avaient un peu oublié que croissance et développement, dans une embryogénèse sans fin chez les végétaux, étaient intimement liés. Les hormones de développement se firent oublier pour laisser place au terme de PHYTOHORMONES. Ce qui posa immédiatement problème dans la question des phases de développement (induction, initiation ou évocation, expression) spatio-temporelles. Où le terme de dédifférenciation vint sur le tapis "objectivement". A notre connaissance, cette dédifférenciation ne fut réellement observée que dans des processus tératologiques (hyperhydricité dans des cals ou des pousses feuillées), qui par ailleurs mirent en évidence la complexité du vrai rôle des hormones (les couples auxinescytokinines et polyamines-éthylène) reliées et conditionnées par les métabolismes primaires et secondaires ... et par les stress.

Nous ne sommes pas certains que les biologistes moléculaires actuels tiennent compte de l'état physiologique général (déterminant dans un processus organogénétique) sous l'effet d'une hormone qu'ils croient spécifique, ni que les séquences de gènes allumés ou éteints soient les mêmes spatio-temporellement. L'ingénierie génétique en a déjà fait les frais (par exemple les tomates au mûrissement retardé mais sans saveur!), mais elle devra ramener des physiologistes-hormonologistes à d'autres réalités que les premiers concepts pourtant difficultueusement émergés.

Mots-clés : phytohormones : concepts ; croissance végétale et développement ; différenciation et dédifférenciation végétales ; totipotence organogénétique ; stress végétal ; cancer végétal

\section{Abstract}

Endocrine glands could not be seen in plants as in animal organisms which, in addition have a blood vascular system. Therefore, the concept of plant hormone emerged later only with researches on photo- and gravi-tropisms. The peculiar perception by the apices and a polar distribution of at least one substance engendering an unilateral growth in the elongation zone of stems or roots. A first active natural migrating substance was identified as 3indolylacetic acid (IAA) and called auxin. Coumpounds with a similar or higher activity on growth, after external application were rapidly synthesized, but also generalize the term "growth substance" for both natural and synthetic products.

Exogenous application of IAA or a synthetic analogue may exert a positive or negative effect on growth, depending on both the concentration used and an apparent different sensitiveness of the treated organ (stem, root), in a S-shaped curve. This led to speak in term of sub- and over-optimal concentrations used. But nobody effectively compared the endogenous contents nor took care of the real penetration of the treated plant material. The auxin-oxidase activity of the treated tissues was also not assessed. Auxin-like compounds were synthesized (for agricultural purposes, for instance), the most powerful appearing to be the seleniated IAA and 2,4-D prepared by chemists from our laboratories. Secondly, it was rapidly observed that natural or synthetic auxin application, normally on cuttings, enhanced or induced adventitious root formation. Thus, the first type of growth substance discovered could in addition also affect a development process, "probably" with associated ingredients into a complex called rhizocaline. Among the subsequent "growth substances" discovered, gibberellin favored particularly flowering, "probably" (again) in a multicomplex denominated florigen. The association of other components with auxin and gibberellin in rhizocaline and in 
florigen were never identified although synergistic or antagonistic effectors might exert actions. Plant physiologists progressively recalled to themselves that embryogenesis in higher plants is never ended (certainly not with the completion of an embryo in a seed) and that quantitative growth and qualitative development were intimately interconnected.

The term PHYTOHORMONES emerged, without similarities with animal hormones. The phenomenons of differentitation and dedifferentiation were rediscussed, taking into account the spatiotemporal phases of growth and development. The conclusion of the most general assumption actually is that phytohormones rarely act alone, but rather in couples, through crosstalkings and signaling pathways, depending on gradients in their concentrations (changing with feeding and environmental factors) along the whole plants, even in connection with primary and secondary metabolisms.

In vitro cultures of calli and vegetative multiplication using sequences of (phyto)hormones (natural and synthetic) in cocktails with minerals and other compounds (carbohydrates) induce traumatology symptoms that help to better distinguish tumors from real plant cancers. The latters also necessitated a specific plant physiological approach. Conscientious that the above considerations might take place in a life science history, where the concept of a plant hormone was distorted by animal physiology, it is hoped that the above reminders will help molecular biologists and genetic engineers to reappraise results on phytohormone specificity.

Keywords : phytohormone concepts; plant growth and development; plant differenciation and dedifferenciation; organogenetic totipotency; plant stress; plant cancer

\section{Introduction}

Les recherches en physiologie animale avaient toujours été privilégiées parce qu'elles servaient de supports à la physiologie humaine et à la médecine. D'emblée, nous oserions dire que la physiologie végétale a comblé son retard et a peut-être à en "réapprendre" à ses alterego de la biologie animale et humaine. N'est-ce pas une des raisons pour lesquelles les cours de Zoologie et de Botanique des débuts de nos études furent réunis plus tard dans un cours de Biologie générale et que plus récemment les deux disciplines font partie d'un seul Département des Sciences de la Vie? La génétique, qui a conduit à la biologie moléculaire, débouchant elle-même à l'actuelle génomique, et au "génie génétique", a-t-elle fait le rapprochement et une certaine unicité? Nous n'avons pas participé nous-mêmes à cette évolution toujours en cours. Nous souhaitons ici expliquer comment nous avons subi l'influence de la physiologie animale sur une hormonologie végétale vécue pratiquement à ses débuts.

Il nous faut sans doute débuter par définir une hormone, tel que cela a été fait d'abord en biologie animale, bien avant de concerner les végétaux. Une hormone est une substance chimique élaborée dans un tissu ou sécrétée par une glande endocrine, agissant à distance par une voie sanguine (ou lymphatique) sur des récepteurs spécifiques de cellules-cibles, avec la particularité de pouvoir intervenir à très faibles doses. En d'autres mots, elle transmet un message sous forme chimique et joue donc un rôle de messager dans l'organisme, exerçant une action spécifique sur le fonctionnement d'un organe par l'intermédiaire d'un processus biochimique. Dans les cours actuels de biologie, elle figure dans les messagers biochimiques. A titre d'exemple, l'hypophyse sécrète en particulier l'hormone de croissance, mais aussi d'autres "stimulines" exerçant un contrôle de la sécrétion d'autres glandes endocrines telles que la thyroïde, le pancréas, les glandes sexuelles, .... Les glandes exocrines déversent généralement leurs produits de sécrétion via des canaux sur la peau ou dans des cavités naturelles communiquant avec le milieu extérieur (glandes sébacées, mammaires, 
digestives,...). La synthèse chimique de nombreuses hormones a été concrétisée, les produits synthétiques s'avérant souvent plus efficaces que les hormones naturelles. Le gain d'efficacité des substances synthétiques par rapport aux hormones (naturelles) continue à croître grâce à une compréhension grandissante des mécanismes d'interaction (avec des synergistes, des agonistes, des antagonistes, des médiateurs...) et des structures détaillées des divers intervenants permettant de gagner en efficacité post-réception. Et il va sans dire que le génie génétique ouvre dans ce domaine de grandes possibilités.

\section{La lente acceptation du concept de phytohormone. En cause: croissance et développement}

Nos botanistes-anatomistes du début n'ont pas pu décrire de véritables glandes, au sens endocrine, et exocrine des animaux, mais les plantes possèdent des poils glanduleux, glandes et cellules sécrétrices souvent à la surface des dépressions de différents organes.

Citons les sécrétions gluantes des plantes carnivores (enzymes), les huiles essentielles des plantes aromatiques. Ils n'imaginaient donc pas d'hormones végétales ciblées d'autant plus que les plantes ne possédaient pas de véritable système circulatoire, même si les deux types de sève avaient très tôt été mis en évidence. Et ils étaient loin d'imaginer des transports inercellulaires via les plasmodesmes ou par le truchement de l'exo-endocytose, les espaces intercellulaires et les parois pecto-cellulosiques. Et nous n'avons surtout assisté à aucun procès fait par des botanistes à des zoologistes ou médecins, enseignant la biologie générale, animale et végétale, qui parlant de l'embryogénèse, clamaient que celle-ci se terminait chez les végétaux par la finalisation de la graine. La dormance, la post-maturation ne sont que des étapes momentanées d'une embryogénèse continue tout au long de la vie de la plante. Même des traités récents de Biologie des Organismes parlent de développement post- embryonnaire (Clos et al. 2006). Nous voulons aussi amener l'idée qu'il est bien difficile chez une plante de distinguer croissance (processus enseigné comme quantitatif) de développement, organogénèse ou morphogénèse (processus qualitatifs), et que cela entraînera un retard de plus et des erreurs de concepts chez les phytophysiologistes.

À titre d'exemple, la première hormone découverte chez les végétaux, ainsi que les suivantes, fut appelée hormone de croissance et les composés synthétiques mimant leurs effets, substances ou facteurs de croissance. Les termes phytohormones $=$ hormones végétales eurent même du mal à s'imposer. Il est vrai que rien ne prouvait, à ces époques de découvertes successives, que ces phytohormones et facteurs de croissance pouvaient agir ou provoquer des processus de morphogénèse aussi à distance. Cela a même amené les phytobiologistes à "patauger" en s'obstinant à chercher ou imaginer des hormones spécifiques de certains développements (rhizocaline pour la rhizogénèse, florigène pour la floraison, par exemples), furent-ils à plusieurs composants (Bernier 1988), sans pouvoir jamais les identifier... dans l'erreur fatale d'oublier -nous le répétons- que l'embryogénèse de la plante est continue, que croissance et développement sont intimement liés ... et ce n'est que récemment qu'on y voit les mêmes phytohormones impliquées, certes de manières différentes, dans les deux types de processus. Cela fait partie de notre objectif ici de souligner cette spécificité supplémentaire des hormones végétales par rapport aux hormones animales.

De nombreux biologistes sortant des "Sciences de la Vie" n'ont pas encore bien perçu cette histoire pour en tirer profit, même dans leurs recherches actuelles. Nous avons pu nous en rendre compte personnellement lors de la lecture, à l'écoute de la présentation de mémoires ou de "reviews" de manuscrits pour des journaux de haut niveau pourtant, tout récemment. Nos professeurs et collègues physiologistes ont brillamment retracé l'histoire des pionniers de la physiologie végétale (Bouillenne 1946; Bernier 2013). En plaçant la première phytohormone découverte, l'auxine, au cœur des processus de croissance et de développement 
ne sont-ils pas "tombés dans le panneau", pas encore conscients cependant que les différentes phytohormones (qu'on dit encore spécifiques) agissent rarement seules; leurs effets résultent bien souvent d'actions coordonnées de plusieurs d'entre elles, peut-être se distinguant encore, d'une autre manière, des hormones animales. Nous savons maintenant par exemple que le contrôle de la différenciation, où les concentrations relatives en auxine sont si importantes, est sous la dépendance de plusieurs protéines membranaires, qui déterminent la distribution de l'hormone dans les tissus (Friml 2003). La biologie moléculaire et la génomique nous apporteront encore d'autres enseignements.

Nous-mêmes avions écrit il y a plusieurs années "Changing concepts in plant hormones action (Gaspar et al. 2003) en oubliant aussi, pour partie, ce qui est dit plus haut. Sommes-nous en train de vouloir rectifier le tir ou d'en rajouter à l'Histoire des Sciences?

\section{La première hormone végétale: l'auxine}

Les botanistes-anatomistes avaient tôt constaté que l'allongement des plantes ne pouvait pas résulter seulement d'une multiplication des cellules par mitoses (mérésis); la constitution de grandes vacuoles aqueuses et l'étirement des parois, deux typicités végétales, participaient à une forte croissance des cellules (auxésis). Dès la fin du $19^{\mathrm{e}}$ siècle, les premiers physiologistes voulurent impliquer des substances (parfois hypothétiques) dans ce processus. Des expérimentations suivies furent entreprises par un pionnier de la Physiologie végétale, Francis Darwin, le fils de Charles Darwin, à la fin du $19^{\text {e }}$ siècle (voir Bouillenne 1946; Bernier 2013), sur les tropismes. Une série de découvertes au début du $20^{\mathrm{e}}$ siècle jetèrent les fondements de l'Hormonologie végétale. Les apex des coléoptiles et plus généralement des tiges furent tenus pour responsables de la perception de la lumière et déterminants pour des élongations s'opérant en dessous d'eux. Il en fut conclu que les apex étaient aussi les sites de production d'une substance mobile, donc d'une hormone translocable, dans ce cas-ci, de manière basipète (polaire) pour agir à distance. Cette substance hormonale fut identifiée, non sans difficulté, à l'acide ß-indolyl acétique (AIA). Et elle fut retrouvée dans de nombreux végétaux. Nos patrons vécurent ces découvertes, participèrent à de nombreuses expérimentations avec leurs collègues, parallèlement à celles qu'ils entreprenaient avec leurs collaborateurs. Des découvertes, dont certains principes sont toujours acceptables actuellement, se succédèrent alors à un rythme accéléré, mais des concepts, pas toujours vérifiés, se répandirent, et faussent sans doute encore des expérimentations d'aujourd'hui.

L'AIA fut considéré comme une hormone présente dans tous les végétaux. Est-ce vraiment le cas? A titre d'exemple, l'AßIA considéré comme AIA dans Lens culinaris par Pilet (1961) fut identifié comme étant l'acide indolyl-ß-acrylique (Hofinger et al. 1970). Il est vraisemblable que l'AIA et AIAcryl co-migraient au même endroit dans les chromatographies simplistes des débuts. Les auxines de synthèse pour des utilisations pratiques (comme herbicides totaux ou sélectifs notamment) dans les années qui suivirent, avaient pourtant édicté des règles à suivre (Deysson 1967) pour s'assurer d'une efficacité certaine et plus marquée (pensons au 2,4-D, au NAA). L'idée a alors prévalu que l'AIA était synthétisé seulement dans les apex et il fut tenu pour seul responsable de nombreuses corrélations observées précédemment (notamment dans le concept de dominance apicale; débourrement de bourgeons sous-jacents à l'apex suite à une décapitation). On sait maintenant que l'auxine est vraisemblablement synthétisée dans toute cellule vivante, y produisant localement plusieurs types d'actions. Les apex sont-ils les seuls à avoir un surplus d'auxine exportable jusque dans les racines, où elle exerce plusieurs contrôles? On a des arguments actuels aussi pour affirmer que l'auxine n'est pas seule en cause dans la dominance apicale.

L'auxine agit-elle sous forme libre ou conjuguée (par exemple sous forme d'AIAaspartate), ou les formes conjuguées ne sont-elles que des formes de réserve d'auxine qui peut 
être libérée à "certaines occasions"? Dans des études récentes, des chercheurs comptabilisent toutes les formes d'auxines détectables et quantifiables par les instruments les plus récents (HPLC, spectrométrie de masse) lorsque l'on croit l'auxine, plutôt les auxines, en jeu, sans pouvoir directement affirmer laquelle mettre en cause dans un processus de croissance ou de développement étudié.

Après sa découverte, l'AIA fut appliqué (à tort et à travers sous diverses formes: solutions, gels) sur différents types d'organes. Dans diverses situations, avec des stimulations de croissance très différentes selon les organes, et on parle encore de sensibilité des racines, des bourgeons, des tiges, comme cela est montré dans la Figure 1 (Gaspar et al. 2003).

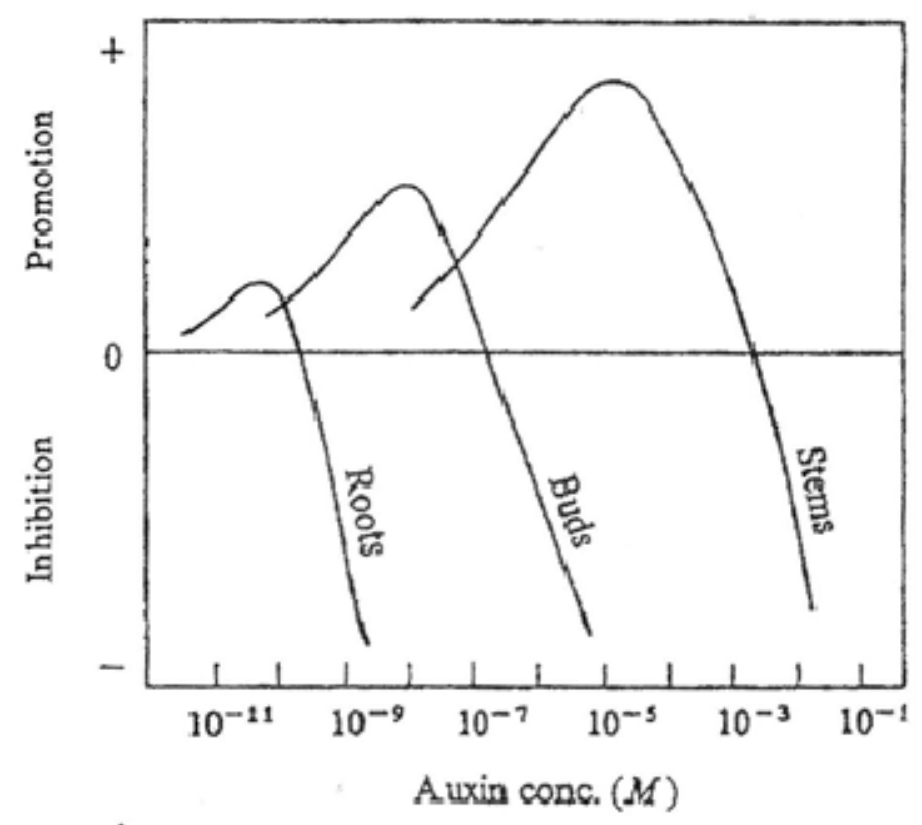

Fig. 1 : Représentation schématique de la croissance des racines, des bourgeons et des tiges en réponses à des concentrations croissantes d'auxine (Thimann, 1937).

S'agit-il vraiment d'une sensibilité différente, au sens d'une réceptivité différente (différences de quantité et de sensibilité des récepteurs, des mécanismes de transmission du signal)? Ces organes ne contiennent-ils pas déjà des quantités différentes d'auxine endogène au départ? La Figure 1 montre bien que l'application d'auxine peut avoir des effets activateurs ou inhibiteurs selon sa concentration et qu'une même concentration peut activer la croissance d'une tige et inhiber celle des racines. On qualifia les doses inhibitrices de sus-optimales ou supraphysiologiques (on retrouvera ces types de courbes en cloche pour toutes les hormones découvertes ultérieurement). Mais tout cela ne tient pas compte de la capacité de ces différents organes à laisser pénétrer l'auxine appliquée, à la transporter, à la métaboliser, etc... N'a-t-on pas été jusqu'à proposer que des composés phénoliques, activateurs ou inhibiteurs de l'activité auxine-oxydasique, étaient les véritables modulateurs de la croissance (Pilet et Gaspar 1968). Et l'AIA-oxydase, parlons-en. Si in vitro l'AIA peut être détruit par pratiquement toutes les "isoperoxydases", certes dans des conditions différentes et par des mécanismes divers, lesquelles sont opérationnelles in situ? Il est à peu près certain que les auxines les plus puissantes, AIA sélénié et 2,4-D sélénié synthétisés à Liège (Hofinger et al. 1980, Tadino et al. 2003) le sont parce qu'elles sont moins dégradables après leur application. Il est acquis en tous cas que les organes "agressés" par une application d'auxine qu'ils jugent sans doute excessive (nous sommes conscients du subjectif) réagissent par une activationadaptation de leur système AIA-oxydasique qui va dans, certains cas, jusqu'à abaisser leur 
taux endogène initial. Est-ce la cause de l'inhibition de croissance causée par cette application d'auxine supra-physiologique? Les mêmes questions se posent à propos du rôle de l'auxine (et des autres hormones identifiées ultérieurement) dans le phénomène de gravitropisme où les apex des racines ont aussi été considérés comme des sites de perception de la gravité.

Dès la fin du $19^{\mathrm{e}}$ siècle, l'hypothèse était que les différents types d'organogénèse résultaient de "Bildungstoffe" présentes en quantités limitées (Bouillenne 1946). L'application d'auxines naturelles ou de synthèse dans la première moitié du siècle suivant montrait un effet promoteur sur la rhizogénèse des boutures. Avait-on trouvé des effets limitants? Il semblait inconcevable que l'auxine seule, déjà impliquée dans les processus de croissance par élongation cellulaire, fut rendue seule responsable du déclenchement d'une organogénèse spécifique, d'autant plus que les effets d'auxines endogènes pouvaient eux-mêmes être modifiés par la présence de sucres, de phénols, et qu'entrait vraisemblablement en jeu une oxydase. C'est avec ces données que Bouillenne et Went (1933) émirent l'hypothèse d'un complexe rhizogène qu'ils dénommèrent RHIZOCALINE. Toutes les tentatives d'isolement et d'identification d'un root forming complex s'avérèrent négatives, mais une auxine était toujours présente. Il fallut admettre que l'hormone agissait en maître aussi bien dans le processus de croissance que de développement, avec des facteurs limitants sans doute différents, mais aussi en intervenant dans les processus de divisions cellulaires (mérésis).

Des recherches récentes ont montré l'implication de l'auxine dans de nombreux autres processus de développement. Outre les racines, l'auxine est essentielle pour la formation des feuilles dans les méristèmes (Reinhardt et al. 2003), des nervures dans les primordia foliaires (Mattsson et al. 2003), et des tissus conducteurs (Ohashi-Ito et al. 2013). Tous ces processus organogènes résultent de l'accumulation d'auxine dans des sites bien précis. Cette accumulation est due à la présence de transporteurs transplasmalemme, dont la localisation au sein des cellules est contrôlée très précisément. C'est aussi ces transporteurs qui sont responsables de la migration basipète de l'auxine du haut en bas de la plante (Petrášek et Friml 2009). Ils assurent également la redistribution de l'AIA dans l'apex racinaire (Friml 2003). La question se pose de savoir quel est leur rôle dans la croissance des cals et dans l'organogénèse que ceux-ci manifestent dans certaines conditions.

\section{Les gibbérellines}

Nos patrons assistèrent un peu plus tard à la découverte des gibbérellines qui "transformaient"

des plantes naines en normales. Le "gigantisme" provoqué par des applications de gibbbérellines à certains stades résultait surtout d'élongations cellulaires. Il ne leur fut pas aisé d'admettre ex abrupto l'existence d'hormones autres que l'auxine. Leur idée première fut que les gibbbérellines n'étaient que des facilitateurs de l'action de l'auxine sur l'extensibilité des parois cellulaires. Certains d'entre nous furent amenés à mener des recherches sur la variation d'auxine endogène après application de gibbérellines comme d'autres le faisaient (réfs. dans Leyh et al. 1963), mais sans résultats immédiatement probants. Nous aboutîmes à constater qu'effectivement les résultats des mesures du taux auxinique après application de gibbbérelline variaient selon le temps écoulé entre l'application et les dosages. En réalité l'application efficace d'une gibbbérelline augmente le taux d'AIA endogène, mais cette augmentation n'est maintenue qu'un certain temps, car apparemment (ce fut notre interprétation) il rechute rapidement après jusqu'à des valeurs inférieures à celles d'avant le traitement.

On l'a vu plus haut, la plante adapte rapidement un de ses systèmes auxineoxydasiques à une augmentation endogène d'un taux d'auxine supérieur à sa "normalité". Ce type de recherche fut sans doute abandonné car progressivement la biologie moléculaire 
prenait le pas avec des recherches sur les récepteurs et les transporteurs de l'auxine et la régulation de gènes particuliers.

L'application exogène de gibbérellines n'avait cependant pas d'effet rhizogène comme les auxines mais elles manifestaient des actions florigènes (provocation de la floraison en conditions environnementales défavorables, avancement en conditions favorables, abondance de la manifestation) mais les arguments évoqués plus haut, notamment la séparation difficile entre croissance et développement, avec d'autres sans doute, amenèrent, entre autres, Chaylakhyan (1985), à proposer l'existence d'un FLORIGÈNE, un complexe florigénique à au moins deux composants: une gibbbérelline et une hypothétique "anthésine" qu'on ne parvint jamais à identifier. La théorie fit long feu. Elle avait surtout ses partisans chez les scientifiques du bloc de l'Est de l'Europe, imprégnés par les idées du maitre à penser de Moscou (sans vouloir déconsidérer qui que soit, mais c'est ainsi que nous le ressentions).

\section{Les cytokinines}

La découverte des cytokinines, leur production d'un surplus exportable de manière acropète à partir de jeunes racines en croissance (il faudra des années pour admettre que pratiquement toute cellule végétale vivante peut produire tous les types d'hormones), et leur contrôle de la croissance des parties aériennes (des végétaux supérieurs en tout cas) mit du temps à faire son chemin. Leurs applications favorisaient surtout les divisions cellulaires (mérésis). Comme pour les auxines, on émit des règles de conformation stéréo-chimique pour qu'une molécule soit considérée comme cytokinine et soit surtout active comme telle. Mais il apparut bientôt que d'autres types de molécules pouvaient mimer leurs effets. Ainsi en fut-il notamment du thidiazuron (Kevers et al. 1991). Skoog et Miller (1954) concluaient qu'auxines et cytokinines étaient synergétiquement requises pour induire divisions cellulaires et croissance, ce qui n'excluait pas des effets antagonistes (en application externe en tout cas) sur le contrôle de la formation des racines et des bourgeons, d'où le schéma classique (Fig. 2) du contrôle du développement par une "balance" entre les auxines et les cytokinines. X

\section{AUXIN(S) CYTOKININ(S)}

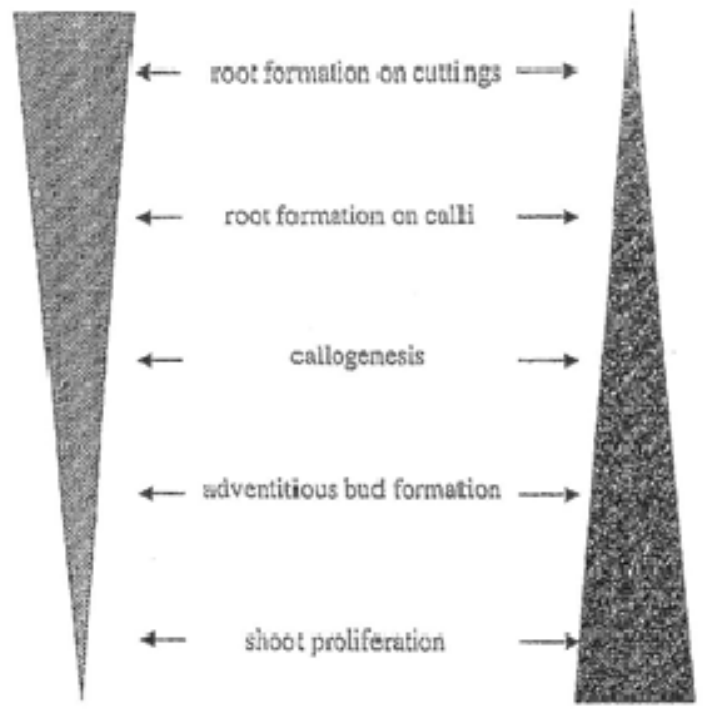

Fig. 2. Contrôle de différents programmes organogènes par la balance entre auxines et cytokinines (repris de Gaspar et al. 2003). 
La découverte ultérieure de messagers secondaires et de "cross-talkings" entre les différents types d'hormone entraînait les chercheurs dans d'autres voies (Gaspar et al. 2003).La découverte ultérieure de messagers secondaires et de "cross-talkings" entre les différents types d'hormone entraînait les chercheurs dans d'autres voies (Gaspar et al. 2003).

Comme des divisions cellulaires précédaient la "transition florale" dans la transformation de méristèmes végétatifs en floraux, celle-ci étant concomitante à des augmentations de cytokinines dans des "exsudats", il n'en fallait pas plus pour attribuer aux cytokinines une "responsabilité" dans "l'évocation florale" et en faire un agent complémentaire dans un florigène à plusieurs composants agissant séquentiellement (Bernier, 1988). Le même auteur, quelques années plus tard, vit l'auxine AIA comme hormone "maîtresse" (Bernier 2013)! Nous avons l'impression que les théories se font au gré des découvertes successives, et que la biologie moléculaire en inspirera encore bien d'autres.

Revenant aux effets spécifiques des auxines et des cytokinines sur le développement, il aura sans doute fallu attendre les cultures de tissus ou d'organes in vitro pour se rendre compte qu'avec ces deux hormones-là, seules, sans se soucier des autres dans un premier temps, on pouvait réaliser les clonages de pratiquement toutes les plantes supérieures; les cytokinines sont nécessaires, sinon indispensables, pour le développemement des bourgeons axillaires et adventifs. Les auxines le sont pour l'enracinement adventif. On ne s'est pas encore trop soucié de savoir exactement si les composés exogènes agissent comme tels de manière endogène, les cellules des explants ou plantules reconstituées "s'arrangeant" elles-mêmes "en interne" pour se procurer d'autres hormones éventuellement nécessaires. Tout juste s'est-on rendu compte que l'éthylène (hormone gazeuse, voir plus loin) émis dans l'atmosphère des flacons par les végétaux cultivés in vitro pouvait influencer croissance et développement, de diverses manières, selon le type container et de confinement (Gaspar et al. 1989; Kevers et al. 1992).

Ces faits nous éclairent mieux maintenant sur les débats sur les termes d'hormones de croissance (sachant bien qu'elles interviennent aussi dans les processus de développement), phytohormones pour certains ou tout simplement régulateurs de croissance (plant growtth regulators) englobant toutes substances intervenant sur croissance ET développement. In fine, nous avons terminé notre carrière avec le sentiment que ces deux processus résultent de gradients variables tout le long du végétal, changeant au gré des modifications d'environnement, de traitements, ... sans oublier les ondes de polarisation-dépolarisation et les gradients électromagnétiques (Desbiez et al. 1987).

Nous fûmes parmi les premiers à montrer qu'une plante en "état floral" (en train de fleurir) pouvait être réversée et poursuivre sa vie en une croissance végétative (Gaspar 1963), par une modification des conditions environnementales réputées "inductrices": personne n'a jamais cherché à réellement mettre en évidence de possibles variations des "gradients" hormonaux.

A force de vouloir disséquer des effets classés (par nous) comme spécifiques, cinquante ans et plus de recherches nous ont amenés à considérer une croissance ET un développement où les différentes composantes (environnementales dont nutritionnelles, hormonales, électromagnétiques...) sont "intégrées", et ce aux phases successives du développement. Nous avons synthétisé ailleurs (Gaspar et al. 2003) les différentes séquences d'événements biochimiques proposés alors et les interactions possibles (dans l'espace et dans le temps) entre des effets de conditions environnementales particulières ou de traitements "spécifiques" sur des développements, la thigmomorphogénèse, par exemple. La littérature récente en propose d'autres sans doute "plus affinés" et plus proches de la vérité. 


\section{Les autres hormones}

L'acide abscissique (ABA) et l'éthylène vinrent s'ajouter aux auxines, cytokinines et gibbbérellines pour constituer les cinq classes alors reconnues comme vraies phytohormones (Gaspar et al. 1996). De nouveaux régulateurs de croissance (et de développement) naturels furent encore découverts: les polyamines, les jasmonates, les brassinostéroïdes, les oligosaccharines, des stérols, des phosphoinositosides, l'acide salicylique, des systémines, l'oxyde d'azote, l'eau oxygénée ... Véritables hormones, messagers secondaires intermédiaires... on ne sait pas vraiment, certains pourtant qu'on est qu'ils interviennent et que l'application de certains, sous certaines conditions, peut provoquer des effets comparables à l'application d'hormones (rhizogénèse par polyamines par exemple). Pour toutes ces substances comme pour les phytohormones, les chercheurs trouvèrent des précurseurs métaboliques, des inhibiteurs ou activateurs de leur anabolisme, catabolisme, conjugaison, transport: pensons à l'acide 2,3,5-tri-iodobenzoïque (TIBA) connu comme anti-auxine et probable inhibiteur du transport de l'hormone, mais dont le mode d'action, à notre connaissance, n'a jamais été complètement élucidé. L'ABA fut généralement considéré comme un inhibiteur de croissance et un antagoniste des actions des gibbérellines (notamment dans l'induction de l'a-amylase dans l'albumen des céréales), mais ceci n'est qu'une question de concentration et les chercheurs oublièrent qu'une même concentration d'hormone peut avoir des effets différents selon la phase de développement et les conditions environnementales (éléments nutritionnels et disponibilité en eau du sol, composition atmosphérique et humidité relative, conditions lumineuses en quantité, qualité et durée journalière). Parallèlement, il fut souvent évoqué des actions auxiniques par une synthèse accrue d'éthylène, mais de nouveau les conditions expérimentales peuvent modifier toutes les "donnes". Fut-il jamais élucidé si les effets de l'éthylène étaient provoqués de l'extérieur après son émission (et éventuelle accumulation) plutôt que de l'intérieur?

Des questions apparentées sont posées à propos des autres régulateurs de croissance et plus particulièrement des polyamines qu'on hésite à classer parmi les phytohormones, vu leurs concentrations généralement élevées. La complexité des interactions entre phytohormones et régulateurs sur différents mécanismes de croissance et de développement, sur leur propre métabolisme d'abord, devient un enjeu pour les biologistes moléculaires s'ajoutant aux erreurs de concepts évoqués en début d'article. Mais cela n'a pas empêché des praticiens de profiter de ces découvertes et de ces interactions pour les utiliser dans des applications pratiques horticoles ou forestières. Citons la synthèse et l'utilisation des "retardateurs de croissance" (notamment CCC et autres) pour contrer la verse des céréales, ou l'inactivation d'un gène impliqué dans la synthèse de l'éthylène, par génie génétique, pour retarder le mûrissement de certains fruits comme la tomate, le melon, etc...

\section{Cycles de croissance et phases de développement sous contrôle hormonal}

Comme celle des animaux, la croissance des plantes prend place par bonds, séparés plus ou moins distinctement par des périodes de ralentissement. Cela fut nommé cycles de croissance : une première allant de l'œuf au développement de la graine, la deuxième débutant avec la germination, jusqu'à l'âge adulte (mais que signifie "adulte" chez les plantes?), la troisième correspondant à la floraison et à la fructification (celle-ci à répétition sur plusieurs années chez les plantes pluriannuelles), une dernière traduite par la mort de l'organisme entier. Chacune de ces étapes peut être décrite par une courbe en S, comme d'ailleurs la forme généralisée de la courbe de croissance d'une plante entière (Fig. 3, reproduite de Bouillenne 1946). Sait-on seulement, à l'heure actuelle, si toutes les hormones précitées interviennent dans chacun de ces cycles? Cette croissance résulte de divisions et d'élongations cellulaires. 
Les mitoses sont aussi impliquées dans les processus de formation des organes. On sait maintenant que le cycle cellulaire est étroitement contrôlé par les phytohormones. Elles modulent la présence des diverses protéines contrôlant le passage d'une étape du cycle à la suivante (Stals et Inzé 2001).

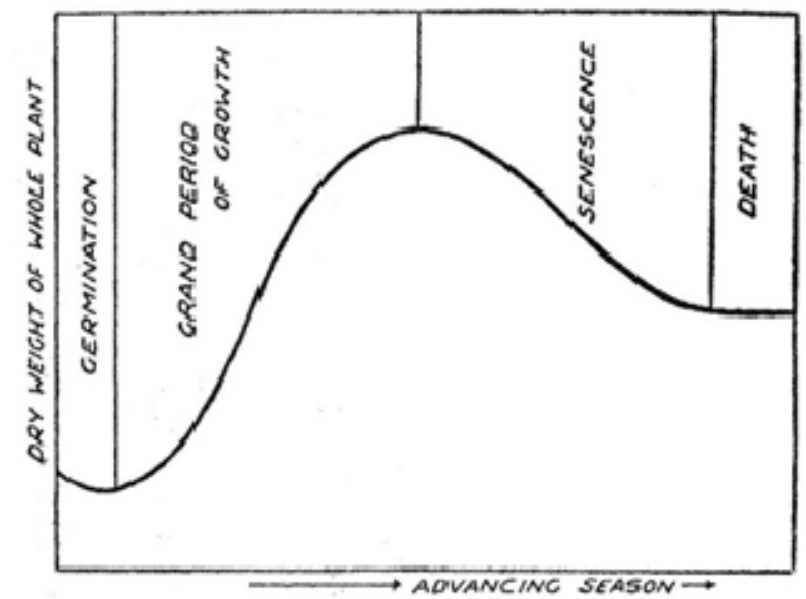

Fig. 3. Forme générale de la courbe de croissance d'une plante, concernant le deuxième cycle allant de la germination à la mort. Cette courbe exprime les changements de poids secs de la plante entière. Les modifications dues à l'apparition de la sexualité (floraison et autres) n'y sont pas représentées (repris de Bouillenne 1946).

On reste intuitivement sur l'idée qu'une hormone ou une autre intervient majoritairement dans tel ou tel processus. La découverte de chacune des phytohormones, en effet, s'est faite fortuitement à l'occasion de recherches plus approfondies sur tel processus de croissance ou de développement; on n'est pas revenu systématiquement sur chacun de ceux-ci pour étudier l'activation séquentielle des gènes dans un contexte d'intégration de plusieurs phytohormones. Il reste du travail à faire. Ces cycles de croissance se doublent de phases spatio-temporelles dans les processus de développement concomitants. La floraison en est un bel exemple avec une induction dans les feuilles, une évocation florale dans les méristèmes jusqu'alors végétatifs, avant le développement de l'appareil floral. Dans l'optique d'une variation de l'auxine endogène dans des tiges et feuilles soumises à l'enracinement, sur un milieu à prédominance hormonale auxinique, généralement in vitro, une recherche systématique de la variation de l'activité peroxydasique (ayant une activité auxineoxydasique) globale dans des extraits bruts (effectuée dans des conditions standardisées) et de leurs isoperoxydases séparées par électrophorèse (nous considérons les plus cathodiques comme les plus actives en tant qu'AIA-oxydases, ayant par ailleurs constaté une intervention plus spécifique des isoperoxydases anioniques dans la lignification), nous amenèrent à décomposer le processus de rhizogénèse adventive d'abord en deux puis en trois phases "organogénétiques". Celles-ci furent dénommées induction, initiation, expression et, curieusement nous amenèrent à conclure que la bouture devait être "vidée" de son auxine préalablement à l'initiation de primordia racinaires (Fig. 4 in Gaspar et al. 1997).

Des traitements autres qu'auxiniques provoquant la rhizogénèse amenèrent les mêmes observations. Les variations étaient d'autant plus accentuées qu'elles concernaient les portions (généralement basales) de boutures néo-formant les racines, mais elles étaient aussi perceptibles tout le long des tiges feuillées. Nous n'avons pas pu déterminer les tissus ou cellules impliquées dans les variations évoquées, mais les recherches confirmèrent d'abord une précédente conclusion: toute la plante participe à un événement organogénétique après 
avoir subi une redistribution générale de son état hormonal. Ceci empêche-t-il une action ciblée d'une auxine sur un gène particulier pour déclencher une séquence particulière d'allumage d'autres gènes dans un "signaling pathway" aboutissant à un message vraiment spécifique comme on le suppose pour telle protéine ou tel ARN, le tant recherché florigène (voir le site internet "flor-id interactive database").

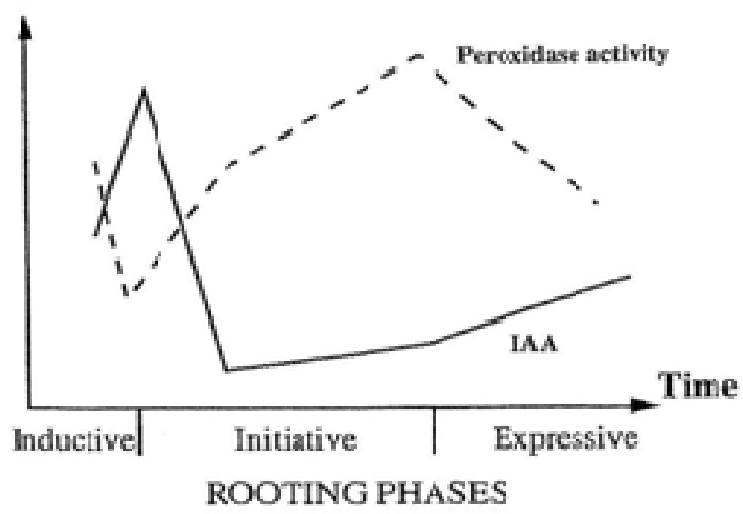

Fig. 4. Repésentation schématique des changements dans l'activité peroxydasique et le taux d'auxine endogène libre, lors des phases successives d'induction, d'initiation et d'expression de la rhizogénèse.

Mais la conclusion la plus étonnante à laquelle nous avons abouti est qu'une bouture lors de son induction à la rhizogénèse passe par une "vidange" (sans doute exercée par une augmentation transitoire des peroxydases) de son contenu auxinique. L'initiation des primordia racinaires qui suit, elle, nécessite une remontée du niveau auxinique.

Nous avons déjà souligné de telles variations d'état physiologique lors de la floraison (Gaspar et al. 1996).

\section{Phytohormones, différenciation et dédifférenciation}

Lorsqu'une élongation plus ou moins importante a pris place dans les cellules issues des méristèmes de tige ou de racine, s'ensuit, si elle n'a pas déjà débuté en cours d'allongement, la différenciation qui aboutira à des cellules spécifiques, regroupées en tissus, qui eux-mêmes se structureront pour former des organes. Destinée de chaque cellule nouvelle et mise en place dans une anatomie caractéristique d'une morphogénèse typique d'un groupe systématique, gouvernée par des gènes homéotiques (Mukherjee et al. 2009) impliquent inévitablement une hérédité génétique. Mais celle-ci doit être guidée par des facteurs physiologiques où indubitablement des phytohormones ont été mises en cause, de manière séquentielle dans des équilibres différents, mais lesquels? Objectivement, il ne peut en être autrement puisque des applications exogènes d'hormones (diverses d'ailleurs) sont capables de détourner des morphogénèses normales, en "obligeant" des tiges à donner naissance à des racines adventives, ou des racines à se doter de bourgeons adventifs.

Ces observations amenèrent logiquement la notion de dédifférenciation. Celle-ci impliquait que des cellules adultes, déjà différenciées, redeviennent juvéniles, autrement dit que non seulement elles reprennent des mitoses mais aussi qu'elles assument une nouvelle destinée tissulaire et organogénétique à leur descendance. Le terme dédifférenciation a caché longtemps une ignorance puisque nous doutons que les précitées "rejuvénilisations" (de l'état cellulaire) et "réorientations" aient jamais été observées et décrites histologiquement. Mais 
ces termes sont encore utilisés maintenant même si certains les remplacent par le terme "induction". Celui-ci est sans doute aussi vague, mais il a le mérite de placer le problème sous un angle physiologique et de plutôt concerner des cellules souches (non encore déterminées) de tissus particuliers.

Le terme de dédifférenciation a souvent été utilisé, par ailleurs, lors de la formation de cals, dits faits de cellules indifférenciées en culture in vitro en particulier à partir de n'importe quel type de fragments d'organes, sous l'effet d'un cocktail composé en général d'une ou des auxines et une ou des cytokinines, le plus souvent à parts égales. La plupart des cals sont en fait constitués de cellules différenciées (bois, liber,.. cela a été observé histologiquement, Crèvecoeur et al. 1987) mais non organisées en tissus. Mais une modification de la balance hormonale à laquelle sont soumis les cals peut les amener à former des bourgeons ou des tiges (cytokinines prépondérantes dans le milieu de culture) ou des racines (auxines favorisées). Des études anatomiques semblent indiquer que ces néo-formations sont issues de cellules restées juvéniles. Ces cals, généralement photosynthétiques, organogènes, sont qualifiés de "normaux". Ils ont sans doute fait avancer le "Schmilblick" de l'interprétation croissancedéveloppement sous contrôle phytohormonal chez les plantes, mais on mesure la somme de questions à solutionner. Les tumeurs végétales (voir Gaspar, 1999), restant organogènes, posent les mêmes types de problèmes, en terme de totipotence.

\section{Perte partielle ou totale de la totipotence organogénétique. Les notions de progression néoplas(t)ique et de cancer}

Si nous sommes persuadés que le génie génétique corrigera la situation existante de non-totipotence organogénétique chez les animaux, sans nécessairement recourir à des cellules souches ou primordiales externes, la totipotence organogénétique chez les végétaux supérieurs en faisait une de leurs caractéristiques exclusives participant à leur éloge (Hallé 1999; Gaspar 2007). La réputée revue Nature refusa en 1998 de faire "reviewer" un de nos articles intitulé "Plants can get cancer" (Gaspar 1999) parce qu'il s'opposait à un article qu'elle avait publié "Why don't plants get cancer" (Doonan et Hunt 1996). Certes les plantes ne véhiculent pas de métastases, mais le terme "métastase" doit-il être la référence pour définir un cancer végétal annonçant une mort de l'organisme? Nous allons y revenir. La mort cellulaire programmée (concernant des cellules isolées ou des tissus) ou apoptose a été reconnue dans les deux règnes (Hasler et al. 2001), même si le terme peut avoir des significations différentes (mort cellulaire réelle, ou participation à une différenciation cellulaire, chez le xylème par exemple). A propos de cancer, la physiologie animale s'est aussi parfois voilé la face en refusant par exemple une hérédité génétique du cancer du sein (les médecins interrogent pourtant ses patientes pour savoir si leur mère en était atteintes) et reportent leur cause sur "l'épigénétique"sous l'effet d'un déséquilibre hormonal naturel ou provoqué par divers médicaments proposés notamment à la ménopause. "Hormonodépendant" sans autre précision est le terme utilisé par les médecins. Dérèglement dans la balance hormonale: nous revoilà au cœur du problème de la spécificité, ou plutôt de la nonspécificité d'une hormone sans tenir compte de la "balance" avec les autres et sans doute d'un gradient à plusieurs composantes le long de tout l'organisme définissant un état physiologique particulier. Nous voudrions dès maintenant réinsister sur la complexité dans la recherche d'un mode d'action spécifique de chacune des phytohormones, sans une intégration dans le contexte d'un état physiologique global déterminé de l'intérieur et surtout par des facteurs environnementaux ou des traitements exogènes chez les végétaux.

Un cal normal, tel que provoqué comme évoqué plus haut par une quantité plus ou moins égale d'une auxine et d'une cytokinine, peut-être sub-cultivé régulièrement pendant plusieurs années en présence des mêmes hormones ou équivalants chimiques tout en gardant sa 
totipotence, c'est-à-dire former des bourgeons se développant en tiges feuillées sous l'effet d'une balance en faveur des cytokinines, ou former uniquement des primordia se développant en racines sous l'effet d'une balance à prépondérance auxinique. L'isolement des tiges feuillées, enracinées ensuite sur un milieu à forte prépondérance auxinique conduira à la reconstitution des plantes entières, parfaitement viables. C'est le principe du clonage à partir des cals; il en existe d'autres à partir de bourgeons apicaux ou axillaires, notamment. Il est arrivé cependant chez des producteurs "industriels" par culture in vitro que de telles plantes, pourtant bien vigoureuses et apparemment normales, à l'état végétatif, ne fleurissent pas en temps attendu, ou pas du tout, en conditions pourtant qualifiées de normales. Dérèglement hormonal? Le comportement de ces plantes traduisait indubitablement une mémoire de traitements vécus, à l'image de pousses feuillées, apparemment identiques et de la même année, qui issues du haut d'un arbre ou de "rejets" à sa base ne réagissent pas de la même manière in vitro pourtant sur des milieux et en conditions environnementales identiques. Les hormones sont donc mises en cause aussi dans cette mémoire. A ce propos, il est intéressant de mentionner le "carry-over" de caractéristiques thigmomorphogénétiques (structurales et biochimiques) dans des cals induits à partir de plantes "irritées" mécaniquement et ayant subi des modifications hormonales (Bourgeade et al. 1989).

Les cals normaux cultivés pendant de nombreuses années sur milieux hormonaux perdent donc progressivement leur "normalité" puisque leur descendance sous forme de plantules sera "affectée" dans son comportement. La réactivité de tels cals à une balance hormonale exogène modifiée va elle-même se révéler de moins en moins efficace. Ils vont par ailleurs laisser apparaître à leur surface des cellules blanchâtres (pratiquement plus de chlorophylle) qui, isolées, vont néanmoins se multiplier sans nécessiter l'apport d'aucun "régulateur de croissance" exogène. Les Anglo-Saxons les qualifièrent de "fully habituated", les Français préférant utiliser le terme de "accoutumance aux hormones" (pourquoi pas à l'absence d'hormones?) sans se poser plus de questions, ou plutôt concluant subjectivement à une "autonomie hormonale". Pourquoi pas, en effet, penser à des "oncogènes" pour la justifier (Hagège et al. 1992)? Dans des cals habitués, nous isolâmes deux lignées, une organogène et une non-organogène, quelles que soient les conditions environnementales ou de milieu de culture imposés. La souche organogène, "self-regenerating" ne produisait, tout en proliférant, que de nombreux bourgeons en surface, quelques rares d'entre eux se développent en minipousses feuillées (blanchâtres) incapables de répondre, isolées, aux auxines pour reconstituer des plantules viables, lorsqu'elles n'étaient pas "décédées" par nécroses préalablement. Dans les deux lignées, les cellules devenaient de plus en plus friables (et sujettes à nécroses) et les pousses éventuelles dans celle qualifiée d'organogène, de plus en plus "breakable". Des analyses systématiques de phytohormones endogènes, par nous et par d'autres, ne purent déboucher sur la conclusion d'une surproduction endogène de l'une ou de plusieurs d'entre elles, sauf peut-être de polyamines (Bisbis et al. 1997).

De nombreuses études de différents métabolismes effectuées sur ces cals par notre Laboratoire en étroite collaboration avec ceux de Genève, Caen et Tours, en particulier, aboutirent à la découverte de modifications du cycle biochimique du carbone, de l'azote, des sucres, des composés tétrapyrroliques qui contribuaient à l'accumulation des polyamines ... comme dans les cellules animales cancéreuses. Il n'en fallut pas plus pour parler de progression néoplasique, et de véritables cellules cancéreuses, aussi chez les végétaux. Les caractéristiques les plus typiques de vraies cellules végétales cancéreuses (à distinguer des tumeurs, Gaspar, 1999) ont été résumées (voir Tableau 1, tiré de Gaspar et al. 1998). La perte irréversible de la totipotence organogén(ét)ique, c'est-à-dire de la capacité pour des cellules végétales de réorganiser des méristèmes primaires organogènes à la fin d'une progression néoplasique (vraisemblablement sous influence hormonale) devint la définition acceptée de "cancéreux" pour des cellules végétales, faute de métastases (Gapar et al. 2000). 
Tableau 1. Caractéristiques indiquant qu'un cal de betterave sucrière, complètement habitué et non-organogène, est composé de cellules véritablement cancéreuses

(en l'absence de pathogènes).

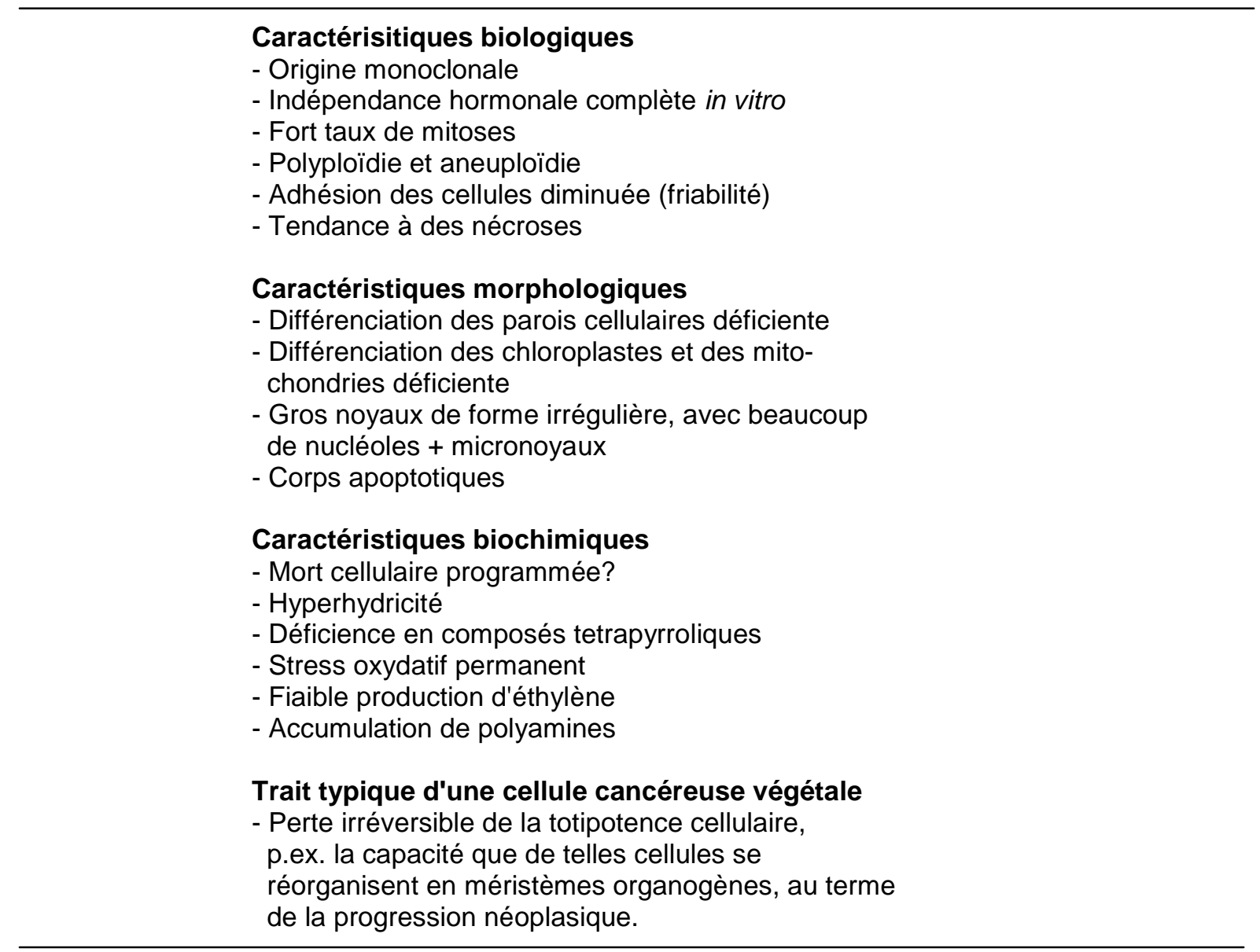

(d'après Gaspar et al. 1998)

10. Les variations phytohormonales endogènes et exogènes parfois victimes d'ellesmêmes, dérivant ou imposant des déviations métaboliques, dont un stress oxydatif permanent irréversible

Les pousses feuillées issues de subcultures répétées in vitro sur des milieux enrichis en cytokinines (en vue du clonage d'une plante-mère, après enracinement) présentent généralement un aspect de plus en plus rajeuni (tiges plus épaisses, moins lignifiées, feuilles plus épanouies), ce qui ajoutait à un concept déjà évoqué dans d'autres circonstances d'une propriété "rejuvénilisante" des cytokinines. Les plantes issues d'un tel clonage bouturaient de manière plus aisée; mais les subcultures de telles pousses sous l'impulsion intempestive d'une succession de "régulateurs de croissance et de développement" (dont les doses minimales pour les buts à atteindre n'ont pas toujours été recherchées) ont quelques fois abouti à la production d'individus "cassants" (entre autres caractéristiques) vitrifiés, mais plus tard qualifiés d'hyperhydriques. Si ces pousses feuillées ne dégénéraient pas directement par nécroses (le plus souvent à partir des apex, les premiers concernés), elles répugnaient alors à 
se faire enraciner sous traitement auxinique. Parce que toutes les phytohormones endogènes ne sont jamais dosées en même temps, il fut difficile dans un premier temps de les caractériser sous cet angle, mais des études détaillées de leurs métabolismes, en relation avec des réactions antioxydatives (Gaspar et al. 2002; Franck et al. 2004) conduisirent vite à un rapprochement entre "habituation" et "vitrification" (hyperhydricité). L'irréversibilité des états illustre bien le schéma de Strasser (Kevers et al. 2004) où la flexibilité du métabolisme est "dépassée" et conduit à une rupture d'homéostasie, un état physiologique et thermodynamique irréversible. Cet état correspond peut-être à des mutations génétiques ou des modifications épigénétiques durables. Notons au passage que ces cals habitués et ces pousses feuillées hyperhydriques, sujettes à nécroses cellulaires (dont des apoptoses, sans doute), outre la perte plus ou moins importante de jonctions cellulaires, présentent de nombreux déficits de différenciation (au niveau des plastes, des membranes, des parois,...). Des éliminations de chloroplastes par autophagie dans des vacuoles auraient même été observées (Franck et al. 1998, 2004), ce qui autoriserait, dans ce cas, l'utilisation du terme dédifférenciation (voir plus haut). Notons aussi que la perte de capacité à former des racines adventives est la première à se manifester (avant celle de bourgeons) dans la progression néoplasique conduisant à "l'abandon" de la totipotence organogénétique dans la cancérisation végétale à l'état cellulaire et à l'état organismique.

Après le constat d'une plus grande quantité de polyamines dans les cellules cancéreuses, des recherches intenses conduisirent à la découverte d'une fixation non-photosynthétique du $\mathrm{CO}_{2}$, une plus faible production et émission d'éthylène, et une synthèse beaucoup moins importante des hémoprotéines, par rapport aux cellules de cals dits normaux. Des déviations de métabolismes du carbone et de l'azote, entraînant des dérives dans d'autres cycles, aboutirent à souligner l'importance du couple polyamines-éthylène, non sans "cross-talkings" avec le couple auxines-cytokinines. Ces interrelations ne firent que traduire un changement d'état physiologique caractérisé par l'autre équilibre thermodynamique dans une situation de stress comme évoqué plus haut (Gaspar et al. 2002). Il aura fallu ces recherches intensives en traumatologie végétale (provoquées par des "hormones" exogènes) pour montrer la complexité du contrôle des métabolismes des vraies phytohormones en interne, leurs relations entre elles, via les métabolismes primaires et secondaires où la biosynthèse des hémoprotéines, des peroxydases en particulier, joue un rôle non négligeable d'intermédiaire (Gaspar et al. 2003). On est donc loin d'une notion de spécificité de chacune des phytohormones .... nous coupant, nous physiologistes, d'une autre génération, sur le rôle plus déterminant peut-être d'un état physiologique général permissif ou prohibitif, favorable ou défavorable aux processus interconnectés de croissance et de développement.

Telle hormone pour tel processus, oui, mais avec la collaboration de l'une ou d'autres dans une situation spatio-temporelle favorable, préalablement installée par les conditions de l'environnement. Tel est le cheminement des concepts en hormonologie végétale que nous avons vécus.

\section{Une conclusion ?}

C'est peut-être qu'il n'y en a jamais de définitive dans les avancées des recherches fondamentales et appliquées. Cette histoire vécue de concepts évoluant dans le domaine de l'hormologie végétale, finalement dans la physiologie de la croissance et du développement chez les plantes, pourrait servir de modèle dans un cours d'Histoire des Sciences, un cours pour lequel à notre arrivée à l'Université, nous nous demandions à qui et à quoi il pouvait servir. Les organismes végétaux génétiquement modifiés, nécessairement changeant des équilibres hormonaux, en tenant compte des avancées encore plus récentes que celles contées 
ici, serviront-ils fondamentalement à poursuivre l'histoire vécue ici et, de manière plus appliquée à expliquer les mutations en cause dans notre monde actuellement surpeuplé?

\section{Remerciements :}

Les auteurs ont apprécié les suggestions pertinentes du Professeur Jacques Dommes et sa relecture du texte. Ils le remercient vivement.

\section{Références}

Bernier, G. The control of floral evocation and morphogenesis. Ann. Rev. Plant Physiol. Plant Mol. Biol. 39 : 175-219, 1988.

Bernier G. Darwin. Un pionnier de la physiologie végétale. Acad. Roy. Belg. Cl. Sc., ed., Bruxelles, 186p., 2013.

Bisbis B., Kevers C., Gaspar Th. Atypical TCA cycle and replenishment in a nonphotosynthetically fully habituated sugarbeet callus overproducing polyamines. Plant Physiol. Biochem. 25: 363-368, 1997.

Bourgeade P., Boyer N., De Jaegher G., Gaspar Th. Carry-over of thigmomorphogenetic characteristics in calli derived from Bryonia dioica internodes. Plant Cell Tissue Organ Culture 19: 199-211, 1989.

Bouillenne R., Photobiologie. Vaillant-Carmanne, Liège et Masson et Cie, Paris, 787p., 1946.

Bouillenne R., Went F. Recherches expérimentales sur la néoformation des racines dans les plantules et les boutures de plantes supérieures. Ann. Jardin Bot. Buitenzorg. 43:1-178, 1933.

Chailakhyan M. Kh. Hormonal regulation of reproductive development in higher plants. Biol. Plant. 27: 292-302, 1985.

Clos J., Coumans M., Coupé M. Biologie des Organismes, vol. 3, Le Développement PostEmbryonnaire Chez les Animaux et les Végétaux. Ellipses, ed. Marketing S.A., Paris, 607p., 2006.

Crèvecoeur M., Kevers C., Greppin H., Gaspar Th. A comparative biochemical and cytological characterization of normal and habituated sugarbeet calli. Biol. Plant. 29: 1-6, 1987.

Desbiez M.O., Gaspar Th., Crouzillat D., Frachisse J.-M., Thellier M. Effect of cotyledonary prickings on growth, ethylene metabolism and peroxidase activity in Bidens pilosus. Plant Physiol. Biochem. 25: 137-143. 1987.

Deysson G. Cours de Botanique, Physiologie et Biologie des Plantes Vasculaires. SEDES ed., 335p., 1967.

Doonan J., Hunt T. Why don't plants get cancer? Nature 380: 481-482, 1996.

Franck Th., Crèvecoeur M., Wüest J., Greppin H., Gaspar Th. Cytological comparison of leaves and stems of Prunus avium L. shoots cultured on a solid MS medium with agar or gelrite. Biochem. Histochem. 73: 32-43, 1998.

Franck Th., Kevers C., Gaspar Th., Dommes J., Deby C., Greimers R., Serteyn D., DebyDupont G. Hyperhydricity of Prunus avium shoots cultured on gelrite: a controlled stress response. Plant Physiol. Biochem. 42: 519-527, 2004.

Friml J. Auxin transport - shaping the plant. Curr. Opin. Plant Biol. 6: 7-12, 2003.

Gaspar Th. Réponses de Salvia splendes aux transferts, à des moments différents, des conditions photopériodiques inductrices de floraison vers des conditions non inductrices. Acad. Roy. Belg. Cl. Sci. 49: 173-180, 1963.

Gaspar Th. Plant can get cancer. Plant Physiol. Biochem. 36: 203-204. 1998. 
Gaspar Th. Tumors, neoplastic progression and cancers in plants. In: Advances in Regulation of Plant Growth and Development (M. Strnad, P. Pec and E. Beck, eds) Peres Cy Publ., Prague, pp 183-192, 1999.

Gaspar Th. A la Recherche du Vivant. Besoin les Uns des Autres. Imprimerie Derouaux Ordina Edit., Liège, 70p., 2007.

Gaspar Th., Bisbis B., Kevers C., Penel C., Greppin H., Le Dily F., Billard J.P., Huault C., Garnier F., Rideau M., Foidart J.M. Atypical metabolisms and biochemical cycles imposing the cancerous state on plant cells. Plant Growth Regul. 24: 135-144. 1998.

Gaspar Th., Franck Th., Bisbis B., Kevers C., Jouve L., Hausman J.F., Dommes J. Concepts in plant stress physiology. Applications to plant tissue cultures. Plant Growth Regul. 37: 263-285, 2002.

Gaspar Th., Kevers C., Bisbis B, Franck Th., Crèvecoeur M., Greppin H., Dommes J. Loss of plant organogenic totipotency in the course of in vitro neoplastic progression. In Vitro Cell. Dev. Biol. - Plant 36: 171-181. 2000.

Gaspar Th., Kevers C., Bouillenne H., Mazière Y., Barbe J.P. Ethylene production in relation to the micropropagation. In: Biochemical and Physiological Aspects of Ethylene Production in Lower and Higher Plants (H. Clijsters, M. De Proft, R. Marcelle, M. Van Poucke, eds) Kluwer Acad. Publ., Dordrecht, pp 303-312, 1989.

Gaspar Th., Kevers C., Faivre-Rampant O., Crèvecoeur M., Penel C., Greppin H., Dommes J. Changing concepts in plant hormone action. In Vitro Cell. Dev. Biol. - Plant 39: 85-106, 2003.

Gaspar Th., Kevers C., Hausman J.F. Indissociable chief factors in the inductive phase of adventitious rooting. In: Biology of Root Formation and Development (A. Altman and J. Waisel, eds) Plenum Press, New York, pp 55-64, 1997.

Gaspar Th., Kevers C., Hausman J.-F., Penel C., Jouve L., Martin-Tanguy J., Aribaud M., Greppin H. Peroxidase as an indissociable factor of auxin and polyamine metabolisms in the induction of rooting and flowering. Proceed. IVth Int. Symposium: Plant Peroxidases. Biochemistry and Physiology. (C. Obinger, U. Berner, R. Ebermann, C. Penel and H. Greppin, eds) Univ. of Geneva, pp 226-234, 1996.

Gaspar Th., Kevers C., Penel C., Greppin H., Reid D., Thorpe T.A. Plant hormones and plant growth regulation in plant tissue cultures. In Vitro Cell. Dev. Biol. - Plant 32: 272-289, 1996.

Gaspar Th., Le Dily F., Billard J.P., Huault C., Bisbis B., Kevers C., Dommes J. Biosynthesis of peroxidases: a role as interplay between primary and hormonal metabolisms. Proceed. VIth Int. Plant Peroxidase Symposium: Plant Peroxidase Biochemistry and Physiology (M. Acosta, J.N. Rodriguez-López, M.A Pedreño, eds) Univ. of Murcia, pp 169-185. 2003.

Hagège D., Andecol Y., Boccara M., Schmitt P., Jeltsh J.M., Barientos E., Signoret J., Gaspar Th. Ras-related protooncogenes are transcripted in leaves and callus from sugarbeet. $\mathrm{J}$. Plant Physiol. 139: 509-511, 1992.

Hallé Fr. Eloge de la Plante. Pour une Nouvelle Biologie. Edit. du Seuil, Paris, 315p. + add., 1999.

Hasler J., Penel C., Gaspar Th., Crèvecoeur M. Mort cellulaire programmée, apoptose, ... et cellules végétales. L'Année Biologique 40:75-95, 2001.

Hofinger M., Gaspar Th., Darimont E. Occurrence, titration and enzymatic degradation of 3(3 indolyl)-acrylic acid in Lens culinaris Med extracts. Phytochem. 9: 1757-1761, 1970.

Hofinger M., Thorpe T.A., Bouchet M., Gaspar Th. Auxin-like activity of (benzo(b)selenienyl -acetic acid). Acta Physiol. Plant. 2: 275-280, 1980.

Kevers C., Boyer N, Courdouroux J.-C., Gaspar. The influence of ethylene on proliferation and growth of rose shoot cultures. Plant Cell Tissue Organ Culture 28: 175-181, 1992. 
Kevers C., Franck Th., Strasser R.J., Dommes J., Gaspar Th. Hyperhydricity of micropropagated shoots: a typical stress-induced change of physiological state. Plant Cell Tissue and Organ Culture 77: 181-191, 2004.

Kevers C., Hausman J.-F., Hagège D., Gaspar Th. Post-effects of thidiazuron on peroxidase activity and rooting of microcuttings of Kalmia latifolia. Saussurea 22: 27-31, 1991.

Leyh C., Gaspar Th., Bouillenne-Walrand M. Nanisme, acide gibbérellique et effecteurs auxine-oxydasiques chez Zea Mays L. Bull. Soc. Roy. Sci. Liège 32: 430-448, 1963.

Mattsson J., Ckurshumova W., Berleth T. Auxin signaling in Arabidopsis leaf vascular development. Plant Physiol. 131: 1327-1339, 2003.

Mukherjee K., Brocchieri L., Bürglin T.R. A comprehensive classification and evolutionary analysis of plant homeobox genes. Mol. Biol. Evol. 12: 2775-2794, 2009.

Ohashi-Ito K., Oguchi M., Kojima M., Sakakibar H., Fukuda F. Auxin-associated initiation of vascular cell differentiation by LONESOME HIGHWAY. Development 140: 765-769, 2013.

Petrášek J., Friml J. Auxin transport routes in plant development. Development 136: 26752688, 2009.

Pilet P.E. Les Phytohormones de Croissance. Masson éd., Paris, 774p., 1961.

Pilet P.E., Gaspar Th. Le Catabolisme Auxinique. Masson et Cie éd. 148p., 1968.

Reinhardt T D., Pesce E.-R., Stieger P., Mandel T., Baltensberger K., Bonnett M., Traas J., Friml J., Kuhlemeier C. Regulation of phyllotaxis by polar auxin transport. Nature 426: 255-260, 2003.

Skoog F. Miller C.O. Chemical regulation of growth and organ formation in plant tissues cultured in vitro. Symp. Soc. Exp. Biol. 11: 118-137, 1957.

Stals H., Inzé D. When plant cells decide to divide. Trends in Plant Sci. 6: 359-364, 2001.

Tadino, V.L.A., Faez J.M., Christiaens L., Kevers C., Gaspar Th., Dommes J. Synthesis and activity of another seleniated auxin: 2,4-dichlorophenylselenoacetic acid. Plant Growth Regul 40: 197-200, 2003.

Thimann, K.V. On the nature of inhibitions caused by auxin. Am. J. Bot. 24: 407-412, 1937. 